\title{
La aplicación del principio de subsidiariedad en el empoderamiento de los pacientes para el cuidado de la salud: el caso de las enfermedades raras en Argentina
}

\author{
Graciela Moya $^{1}$
}

\begin{abstract}
Resumen: El principio de subsidiariedad, ampliamente definido en filosofía política y social, se refiere no solo a la relación de las comunidades entre sí, sino también a las relaciones del individuo con cualquier tipo de comunidad social. La subsidiariedad, por medio de la participación, promueve la dignidad de la persona, como ser individual y social, y reconoce como primario al bien de la persona individual. Según este principio, los individuos o comunidades predominantes adoptan una actitud de ayuda — apoyo, promoción y respeto — respecto de las menores. Adaptado, puede ser utilizado como un parámetro para comprender el concepto de empoderamiento de los pacientes para el cuidado de la salud. Para que este proceso pueda establecerse se requiere, por un lado, una relación médico-paciente, centrada en el paciente y su familia, en la que reciban las herramientas necesarias para la toma de decisiones en salud y, por otro, se creen entidades sociales intermedias en las que los pacientes y sus familias puedan desarrollar estrategias que permitan mejorar el cuidado de salud. El objetivo de este trabajo es profundizar en este principio y ofrecer un marco bioético que facilite la comprensión y desarrollo del concepto de empoderamiento en salud.
\end{abstract}

Palabras clave: principio de subsidiariedad, empoderamiento para la salud, enfermedades raras

The application of the subsidiary principle for patients' empowerment for health care: the case of rare diseases in Argentina

\begin{abstract}
The subsidiary principle, broadly defined in political and social philosophy, refers not only to the relations between communities, but also to relations of the individual with any type of social community. The subsidiary principle promotes the dignity of persons as social and individual beings by participating and it recognizes as primary the well being of the individual person. According to this principle, individuals or main communities adopt an attitude of help -support, promotion and respect- with respect to minor ones. Adapted, it can be used as parameter to understand the concept of patients' empowerment for health care. In order to establish this process it is required, on one hand, a physician-patient relationship center in the patient and his/her family, in which they will receive the necessary tools for health care decisions and, on the other hand, the creation of intermediate social entities in which patients and their families may develop strategies for health care enhancement. The goal of this study is to reflect on this principle and to offer a bioethical framework to facilitate understanding and development of the concept of empowerment for health care.
\end{abstract}

Key Words: subsidiary principle, empowerment for health care, rare diseases

\section{A aplicaçáo do princípio de subsidiariedade no empoderamento dos pacientes para o cuidado da saúde: o caso das enfermidades raras na Argentina}

Resumo: O princípio de subsidiariedade, amplamente definido em filosofia política e social, se refere não somente na relaçáa das comunidades entre si, senão também nas relaçóes do indivíduo com qualquer tipo de comunidade social. A subsidiariedade, por meio da participação, promove a dignidade da pessoa como ser individual e social, e reconhece como primário ao bem da pessoa individual. Segundo este princípio, os indivíduos ou comunidades predominantes adotam uma atitude de ajuda — apoio, promoção e respeito— referente às minorias. Adaptado, pode ser utilizado como um parâmetro para compreender o conceito de empoderamento dos pacientes para o cuidado da saúde. Para que este processo possa ser estabelecido se requer, por um lado, uma relação médico-paciente centrada no paciente e na sua família, na qual recebem as ferramentas necessárias para a tomada de decisóes em saúde e, por outro, se criam entidades sociais intermediárias nas quais os pacientes e suas famílias podem desenvolver estratégias que permitam melhorar o cuidado à saúde. O objetivo deste trabalho é aprofundar-se neste princípio e oferecer um marco bioético que facilite a compreensão e o desenvolvimento do conceito de empoderamento em saúde.

Palavras-chave: princípio de subsidiariedade, empoderamento para a saúde, enfermidades raras

\footnotetext{
${ }^{1}$ Médica genetista, Máster en Biología Molecular Médica, Máster en bioética. Profesora del Instituto de Bioética, Facultad de Ciencias Médicas, Pontificia Universidad Católica Argentina, Argentina

Correspondencia: gracielamoya@uca.edu.ar
} 


\section{Principio de subsidiariedad: definición y ori- gen}

Si bien el principio de subsidiariedad es aplicado frecuentemente en áreas seculares relacionadas con la filosofía política, la filosofía social, la economía y la empresa(1), fue desarrollado inicialmente por Santo Tomás de Aquino, retomado por pensadores del catolicismo europeo de fines del siglo XIX y principios del XX, consolidado en la encíclica papal Cuadragésimo Anno y definido como uno de los principios directrices de la Doctrina Social de la Iglesia Católica.

La Doctrina Social lo considera como un principio ordenador de la sociedad de carácter normativo. Este principio, junto con el principio cardinal de dignidad de la persona humana -interpretado como fundamento de toda la enseńanza-, del bien común y de la solidaridad $(2, n .160)$, sostiene una visión antropológica fundada en el concepto de persona como ser individual y social, es decir, dueña de sí misma y responsable de sus actos y con la tendencia natural a vivir en sociedad. Tiene un carácter general y fundamental referido al orden de la realidad social en su conjunto $(2, n .161)$, que influye asimismo en la vida política y económica, y a partir del cual es posible fundamentar y justificar "los criterios de discernimiento y de guía para la acción social en todos los ámbitos" (2, n.161).

Desde el punto de vista etimológico(3), subsidiariedad deriva del término latino subsidium. Este se aplicaba en el ámbito militar y se refería a la tercera línea de los soldados romanos, Triarii o los triarios, que solo ingresaban a la batalla si las dos líneas frontales eran insuficientes. A partir de este concepto, subsidium tomó un significado más general de "apoyo útil" o "uso como asistencia", y se transformó en un término que describe un tipo de apoyo que no sería necesario en condiciones ideales.

Si bien, este principio ha sido aplicado desde larga data(4), comienza a desarrollarse en la primera gran encíclica social Rerum Novarum (1891) del Papa León XIII(5), carta abierta a los obispos que instruye una doctrina social sobre las condiciones del trabajo y la colaboración entre la clase trabajadora, las empresas, el Estado y la Iglesia, y propo- ne una organización socioeconómica de cooperación entre las partes. El Papa expresa su apoyo al derecho laboral de formar uniones o sindicatos y al derecho a la propiedad privada del trabajador. En el documento hace referencia constante al respeto por los derechos humanos y a la misión de servicio de los gobernantes, basados en el reconocimiento absoluto de la dignidad humana.

Este documento es la referencia para la encíclica social del Papa Pío XI Quadragésimo Anno (1931), que conmemorara el cuadragésimo aniversario de la anterior, en la que se define públicamente el concepto de subsidiariedad, más específicamente en la relación de los gobiernos con la actividad económica: “...como no se puede quitar a los individuos y dar a la comunidad lo que ellos pueden realizar con su propio esfuerzo e industria, así tampoco es justo, constituyendo un grave perjuicio y perturbación del recto orden, quitar a las comunidades menores e inferiores lo que ellas pueden hacer y proporcionar y dárselo a una sociedad mayor y más elevada, ya que toda acción de la sociedad, por su propia fuerza y naturaleza, debe prestar ayuda a los miembros del cuerpo social, pero no destruirlos y absorberlos" $(6, n .79)$.

El Papa Juan Pablo II, en la celebración del centenario de Rerum Novarum, hace pública la encíclica Centessimus Anno(7) (1991), en la que profundiza este concepto en los campos de la actividad económica y los problemas relacionados con el bienestar del Estado(8): "Una estructura social de orden superior no debe interferir en la vida interna de un grupo social de orden inferior, privándola de sus competencias, sino que, más bien, debe sostenerla en caso de necesidad y ayudarla a coordinar su acción con la de los demás componentes sociales" $(7, n .48)$.

Este principio sustenta dos ideas principales: (a) una estructura mayor y más extensa no debe ejercer funciones que pueden ser llevadas a cabo eficientemente por una menor y más pequeña, y (b) la primera estructura debe apoyar a la segunda y ayudarla a coordinar su actividad con las de la comunidad como un todo, sin interferir con ella(9). La subsidiariedad abarca clásicamente tres subprincipios que se interrelacionan, con aspectos positivos y negativos(3): 
- Sub1 Regla de la asistencia: versión conceptualmente positiva. Expresa que una institución o entidad social superior (aun la sociedad o el Estado) deben sostener a una inferior cuando el individuo o la institución menor no puedan desarrollar sus tareas por sí misma.

- Sub2 Prohibición de interferencia: es la formulación negativa del principio. Prohíbe que una entidad social mayor interfiera con los asuntos de una entidad menor de personas afectadas o de una persona.

- Sub3 Ayudar a otros a ayudarse a sí mismos: es el tipo y alcance del apoyo que se puede considerar razonable.

Su implementación tiene como finalidad que la entidad o institución política o social más pequeńa reciba prioridad y esto se aplique en todos los niveles de la coexistencia humana; por ejemplo, el individuo debe ubicarse antes de la comunidad, la comunidad antes que el Estado, el Estado antes de la federación y así sucesivamente(3). Por ello se aplica de dos maneras, tanto en la relación del individuo con la comunidad como en la relación entre los niveles altos y bajos de organización en una estructura social más compleja(8).

\section{Subsidiariedad vertical y horizontal}

La subsidiariedad puede clasificarse en vertical y horizontal desde una perspectiva aplicada(4).

- Vertical: La forma más popularmente reconocida. Se refiere a la correcta relación entre varios niveles de competencia y soberanía institucional: en el ámbito del poder público (autoridad local, regional, nacional, supranacional — de menor a mayor complejidad y responsabilidad-) los niveles más altos no deben reemplazar a los más bajos, sino ayudarlos, comenzando desde las autoridades locales hacia las supernacionales. La institución mayor protege a la menor en dos sentidos: (a) como intervención activa y soporte, cuando la institución menor no puede por sí misma garantizar la adecuada continuidad de su propuesta social, y (b) como garantía de autonomía de la institución menor cuando el objetivo ha sido cumplido con los recursos presentes en ese nivel.

- Horizontal: Se refiere a compartir competencias, funciones y servicios entre las personas y las entidades sociales y a reconocer la prioridad de la sociedad y cuerpos intermedios por sobre el Estado, fomentando la oportunidad de crear nuevas interacciones, más allá del Estado. La razón de ser de un cuerpo público es la de optimizar los recursos de la sociedad y su habilidad para proveer respuestas a las necesidades de cada uno. El Estado existe no para reemplazar a la sociedad, sino para llevar funciones públicas o proveer apoyo solo cuando la sociedad por sí misma no es capaz de hacerlo. En esta perspectiva, los cuerpos sociales intermedios, entre los ciudadanos individuales y las instituciones públicas, desde la familia hasta las asociaciones, desde las firmas corporativas a las organizaciones sin fines de lucro, adquieren un nuevo rol y significado, haciendo un cambio en el concepto moderno de Estado(4).

Este principio es interpretado y aplicado frecuentemente como un parámetro de referencia al funcionamiento y organización de diferentes instituciones empresariales, sociales y gubernamentales. En 1992 el concepto de subsidiariedad fue adoptado como un principio estructural en la construcción de la Unión Política Europea, como un punto central en el Tratado de la Unión Europea firmado en Maastricht(4), el cual se considera con estatus de ley pública y constitucional. En 2002, en la Convención sobre el Futuro de Europa, el Dr. Heinrich Hoffschulte, primer vicepresidente del Council of European Municipalities and Regions (CEMR), sostuvo que el concepto de subsidiariedad se basa en el principio de proximidad al ciudadano(10), el cual es enfatizado en el Artículo 1 del Tratado de la Unión Europea, por el que "las decisiones serán tomadas de la forma más abierta y próxima a los ciudadanos que sea posible" (11).

Pero estas aplicaciones en otros contextos pueden afectar el significado del término, ya que esta concepción puede tornarse restrictiva respecto de su origen y naturaleza antropológica o filosófica(4). Este principio debe basarse en los conceptos éticos que lo sostienen, como el respeto por la dignidad 
de la persona humana, la promoción de la libertad y responsabilidad de los actos, la aceptación de la diversidad y la contribución de la actividad humana al servicio del bien común y, por ende, de la persona humana.

\section{Aplicación práctica del principio de subsidia- riedad en el área de salud}

Sgreccia define el principio de subsidiariedad en el área de la salud como el principio "por el cual la comunidad, por una parte, debe ayudar más allá donde es mayor la necesidad — cuidar más a quien está más necesitado de cuidados, y gastar más con quien está más enfermo- - , por otra, no debe suplantar o sustituir la libre iniciativa de los particulares o de grupos, sino garantizar su funcionamiento"(12).

La subsidiariedad reconoce que el bien de la persona individual es primario en todas las argumentaciones referidas a las organizaciones políticas y sociales, y que las personas solo pueden vivir en comunidades, que tienen también sus bienes específicos y ejercen el poder por derecho propio(8). Por ello, este principio no puede interpretarse en forma aislada del principio de socialidad y del bien común.

El principio de socialidad hace referencia a la necesidad de la persona humana de vivir en sociedad, como característica natural, ya que solo así se realiza a sí misma participando en la realización de sus semejantes, en la búsqueda del bien común. Sgreccia(12) lo define como el compromiso de todas y cada una de las personas en su propia realización, al participar de la realización del bien de sus semejantes. En este aspecto se complementa con el principio de subsidiariedad: "una estructura social de orden superior no debe interferir en la vida interna de un grupo social de orden inferior, privándole de sus competencias, sino que más bien debe sostenerle en caso de necesidad y ayudarle a coordinar su acción con la de los demás componentes sociales, con miras al bien común" $(2, n .1883)$.

La socialidad tiene como objetivo lograr el "bien común" a través de la consideración de los "bienes individuales", existiendo circunstancias en que las personas requieren del apoyo de la sociedad para alcanzar sus bienes individuales. En la concepción de Fridolin Utz, el bien común es un bien inmanente a los miembros de la comunidad, siendo el punto de partida para determinar la relación entre el individuo y la comunidad(13). El bien común de todos los miembros de la comunidad es el conjunto - no la suma - del bien inmanente de todos y cada uno, ya que es conseguido solidariamente, a través de su participación, y poseído por cada persona como parte de un todo.

\section{El principio de subsidiariedad: el empodera- miento del cuidado de la salud}

El empoderamiento en el cuidado de la salud se interpreta como un proceso social, cultural, psicológico o político, mediante el cual los individuos y los grupos sociales son capaces de expresar sus necesidades, plantear sus preocupaciones, diseñar estrategias de participación en la toma de decisiones y llevar a cabo acciones políticas, sociales y culturales para hacer frente a sus necesidades(14). Este movimiento puede aplicarse como empoderamiento individual, por el cual la persona debe adquirir la capacidad de tomar decisiones y ejercer control sobre el cuidado de su salud. Se entiende en un doble sentido: (a) como proceso por el cual la persona genera estrategias que le permita tomar decisiones autónomas en el cuidado de su salud, y (b) como el resultado de aquellas acciones por las que accede a los beneficios generados de aquellas acciones autónomas(15). Es definido como una acción orientada con foco en la remoción de barreras formales e informales y en la transformación de la relación entre las comunidades e instituciones (15).

O bien, puede aplicarse como empoderamiento para la salud de la comunidad, mediante el cual los individuos actúan en forma colectiva con el fin de conseguir una mayor influencia y control sobre los determinantes de salud y calidad de vida de la comunidad, y legitimar sus decisiones en el área de política de salud(16). Uno de los medios para lograr este objetivo es organizar instituciones intermedias en las que las personas apliquen sus habilidades y recursos en esfuerzos colectivos, con el fin de abordar las prioridades sanitarias y satisfacer sus necesidades sanitarias respectivas (14). Esto genera en los pacientes la necesidad de organizar sus actividades y de proponer directrices 
que mejoren el cuidado de salud, porque, aunque las personas tengan una vida con mayores necesidades en el cuidado de salud, todos tienen algo único y valioso que ofrecer a su comunidad(2).

El principio de subsidiariedad puede considerarse como marco bioético para el desarrollo de instituciones sociales intermedias, consideradas como instituciones ordenadoras especiales(2), ya que permite la incorporación de personas cuyas experiencias particulares ofrecen una perspectiva diferente en la organización de las asociaciones. Tal es el caso de las asociaciones que agrupan a familias con integrantes diagnosticados como portadores de enfermedades raras ${ }^{2}$.

\section{La aplicación del principio de subsidiariedad en el caso de las llamadas enfermedades raras}

Las enfermedades raras son habitualmente crónicas, progresivas, degenerativas, discapacitantes y tienen alta tasa de mortalidad(17). Poseen una complejidad clínica tal que hace difícil su reconocimiento y diagnóstico. Como la mayoría de las enfermedades crónicas, se asocian con una marcada reducción de la calidad de vida individual y del potencial socioeconómico de la persona y su familia.

Aunque ellas tengan distintas manifestaciones clínicas, causas y pronósticos, comparten similares problemas médicos y sociales relacionados con: (a) ausencia o retraso en el diagnóstico e información, (b) ausencia o retraso en el acceso a tratamiento, (c) escasa orientación médica, seguimiento y experiencia profesional, (d) soledad, falta de comunicación entre los pacientes, el médico e investigadores, (e) impacto devastador en el paciente y su familia, (f) dificultad en el acceso a la educación, empleo e inclusión social(18), y (g) escasos proyectos de investigación en fármacos

\footnotetext{
${ }^{2}$ Las enfermedades raras son un grupo amplio de alrededor de 6.000 patologías diferentes de las que aún no se dispone de una clasificación adecuada. Alrededor del $90 \%$ son enfermedades determinadas genéticamente, otras pueden ser causadas por infecciones virales o bacterianas, alergias o de origen ambiental, o con base degenerativa o proliferativa. Estos desórdenes pueden potencialmente afectar a todos los órganos del organismo, y a pacientes de cualquier edad, nińos o adultos, y se caracterizan por su baja prevalencia a nivel mundial. El Instituto Nacional de Salud de Estados Unidos considera como enfermedad rara aquella que afecte al menos a 200.000 personas en ese país. La regulación de drogas huérfanas de la Unión Europea (EU) define una enfermedad como rara si afecta al menos a 5 cada 10.000 ciudadanos.
}

para tratamiento de enfermedades raras(19).

En este complicado contexto, los pacientes y sus familias tienden a formar grupos de personas que sufren de los mismos problemas. No solo para hacer frente a las consecuencias adversas de su enfermedad, en sus implicancias físicas, emocionales y sociales, sino también para negarse a las presiones ocasionadas por los sistemas públicos de salud y las limitaciones que sistemas administrativos de terceros impliquen en la práctica médica(20). No siempre los servicios de salud requeridos por pacientes con enfermedades poco frecuentes están disponibles adecuadamente o no están cubiertos en los hospitales del sistema de salud pública (psicoterapia, terapia ocupacional, rehabilitación, entre otros). Los sistemas de medicina prepagada tienen clara la asistencia a pacientes con patologías comunes, pero muchas veces desconocen las necesidades de patologías poco frecuentes y no son lo suficientemente flexibles para adaptarse a las necesidades de los pacientes y sus familias(21). Esto genera errores o retraso en el diagnóstico e inequidad y dificultad en el acceso al tratamiento o rehabilitación y cuidado. Las instituciones intermedias se vuelven una necesidad para los pacientes con enfermedades raras, porque les permiten coordinar sus esfuerzos, los sus familias, investigadores, servicios y políticas de salud, con el fin de desarrollar estrategias que definan más adecuadamente los protocolos de investigación y el cuidado médico.

Este esfuerzo conjunto permite el desarrollo de organizaciones como EURORDIS(21) en la Unión Europea, NORD(22) en Estados Unidos, Fundación GEISER(18) en Latinoamérica (Grupo de Enlace, Investigación y Soporte de Enfermedades Raras), y portales como ORPHANET(23). Todas ellas se definen como organización "paraguas", es decir que engloban a otras organizaciones más pequeñas, específicas de pacientes y también individuos activos en el área de las enfermedades raras, de manera de construir una fuerte comunidad federal(23), pan-europea(22) o latinoamerica$\mathrm{na}(18)$ de "organizaciones de personas que viven con enfermedades raras para articular sus fuerzas, para potenciar su voz y cuyas necesidades encuentren un espacio de resolución"(18). Estas asociaciones tienen como objetivo contribuir en dos áreas principales: por un lado, para la opti- 
mización de los métodos diagnósticos, los lineamientos de la investigación, el adecuado cuidado médico y tratamiento, y, por otro, la inserción social y cultural de los pacientes con enfermedades raras. La misión que definen estas asociaciones consiste en luchar contra el impacto de las enfermedades raras en sus vidas, para que los pacientes "vivan más, vivan mejor, vivan con sus seres queridos y disfrutando de su comunidad"(18).

La participación de los pacientes y sus familias, junto con el personal de salud, en las instituciones intermedias es una consecuencia característica de la subsidiariedad(2). La subsidiariedad, entendida como principio ordenador social, facilita la participación de las personas que ejercen su autonomía y, a su vez, permite la inclusión de personas vulnerables o con autonomía disminuida por su propia patología. La participación se define como una serie de actividades mediante las cuales el ciudadano, como individuo o asociado a otros, directamente o por medio de los propios representantes, contribuye a la vida cultural, económica, política y social de la comunidad civil a la que pertenece(2). En el caso de estas asociaciones paraguas para las personas con enfermedades raras, participan contribuyendo en la optimización de los diagnósticos, investigación, cuidado médico, tratamiento e inserción social de los pacientes. La subsidiariedad facilita el establecimiento y desarrollo de valores como la promoción de la salud, respeto por los derechos, equidad y justicia social en las comunidades, a través del reconocimiento de su enfermedad, la educación y el derecho a involucrarse en los cuidados de su salud. La participación es considerada como una necesidad moral de hacer frente a la adversidad y se entiende como un acto que todos tienden a cumplir consciente, voluntaria y responsablemente, y que tiene al bien común como objetivo(2). Esta necesidad moral también concierne a la acción del Estado y de los demás poderes públicos, quienes deben crear situaciones favorables al libre ejercicio de la actividad autónoma, inspirados en el principio de solidaridad, para establecer los límites a las facultades de las partes y así proteger al más vulnerable(2).

\section{Conclusiones}

El marco bioético para el desarrollo del movimiento de empoderamiento de los pacientes, tan- to en el ámbito personal como en el de la comunidad, puede sostenerse en el principio de subsidiariedad, cumpliéndose sus tres subprincipios: (a) regla de la asistencia, (b) prohibición de interferencia y (c) ayudar a otros a ayudarse a si mismos. En el empoderamiento individual para la salud, el médico, como integrante de una institución superior, por ser el poseedor de los conocimientos y quien dispone de las herramientas para el cuidado de la salud, debe colaborar en los tres sentidos con su paciente y la familia en el momento de la toma de decisiones. En el empoderamiento de la comunidad para la salud, el gobierno, las instituciones intermedias, la academia y la industria(18), como instituciones mayores, deben sostener, ayudar y respetar la coordinación de las acciones de las instituciones menores o pacientes individuales.

Las asociaciones de pacientes que luchan contra el impacto de las enfermedades raras plantean como misión que los pacientes tengan una mejor calidad y tiempo de vida y lo hagan con su familia y dentro de su comunidad(18). El movimiento de empoderamiento de la comunidad para la salud puede entenderse de manera que el bien propio de un individuo puede solamente ser alcanzado por su adaptación al orden social existente y por el fiel desempeño de las funciones asignadas a esa persona(24). El bien común se interpreta como la prosperidad de todos los miembros de la comunidad, el conjunto de la inmanente prosperidad de todos y cada uno. Para alcanzar esta prosperidad, como objetivo del empoderamiento para la salud, es necesario, por un lado, ayudar más donde es mayor la necesidad — cuidar más a quien está más necesitado de cuidados, y gastar más con quien está más enfermo- $-y$, por otro, no suplantar o sustituir la libre iniciativa de los particulares o de grupos, sino garantizar su funcionamiento(12). Los profesionales de salud tienen que promover en sus pacientes el ejercicio de su capacidad de ver sus dificultades y reconocer sus propias necesidades. El empoderamiento contribuye a que el paciente y su familia ejerzan su autonomía en la toma decisiones en el cuidado de su salud, pero requiere también una mayor participación y responsabilidad de las personas para tomar las mejores decisiones para sí y para su comunidad. Esta participación no debe entenderse en forma aislada de las políticas sanitarias. El gobierno debe 
facilitar el desarrollo de estructuras intermedias, promover el acceso a la información de las comunidades acerca de la prevención y tratamiento de las enfermedades y orientar la práctica médica centrada en la capacitación del paciente para la toma de decisiones, tanto en la promoción de la salud como en la aceptación y la planificación de su vida desde la enfermedad(25).

La aplicación del principio de subsidiariedad en el empoderamiento de los pacientes permite la integración de las necesidades personales y de las comunidades en el cuidado de la salud a través de la participación. Este concepto de subsidiariedad a través de la participación no debe perder su naturaleza antropológica y considerarse como un movimiento social o empresarial. El concepto de subsidiariedad surge desde una visión antropológica del hombre bien precisa y debe asentarse en el respeto por la vida humana, su dignidad inherente, su autonomía y el compromiso de cada una de las personas de participar continuamente en la contribución del bien común y de la persona humana.

\section{Referencias}

1. Melé D. The principle of subsidiarity in organizations. A case study. WP $\mathrm{N}^{\circ} 5662004$ IESE Business School Universidad de Navarra. [online] 2010 [cited 2010 dic 17] Available from: URL: http://papers.ssrn.com/sol3/papers.cfm?abstract_id=884395

2. Pontificio Consejo Justicia y Paz. Compendio de la Doctrina Social de la Iglesia. Buenos Aires: Conferencia Episcopal Argentina, Oficina del Libro; 2005.

3. Gosepath S. The Principle of Subsidiarity. In: Follesdal A, Pogge YT. Real World Justice. The Netherlands: Springer; 2005: 157-170.

4. Istituto Regionale di Recerca della Lombardía. Subsidiarity, Brief Anthology. Regione Lombardia: Arti Grafico Fiorin, San Giuliano Milanese [online] 2009 [cited 2010 dic 17] Available from: URL: http://irer.org/convegni/archivioeventi/eventi-2009/assise-della-sussidiarieta/sussidiarieta-subsidiarity-subsidiarite/subsidiarity.-brief-anthology/

5. León XIII. Carta Encíclica Rerum Novarum. Sobre la situación de los obreros. 15 de mayo de 1891, Pío XI.

6. Carta Encíclica Quadragessimo Anno. Sobre la restauración del orden social en perfecta conformidad con la ley evangélica, al celebrarse el 40 aniversario de la Encíclica "Rerum Novarum" de León XIII, 15 de mayo de 1931.

7. Juan Pablo II. Carta Encíclica Centesimus Annus. En el centenario de la Rerum Novarum, 1 de mayo de 1991.

8. Murray A. The Principle of Subsidiarity and the Church. The Australasian Catholic Record 1995; 72: $163-172$.

9. Catecismo de la Iglesia Católica. Tercera parte la vida en Cristo. Primera sección. La vocación del hombre: la vida en el espíritu. capítulo segundo: La comunidad humana. Artículo 1: la persona y la sociedad. I. Carácter comunitario de la vocación humana. 1883 Madrid: Conferencia Episcopal Argentina; 1993.

10. Hoffschulte H. Working Group I on the Principle of Subsidiariety. Brussels: The European Convention CEMR Position Paper, The secretariat; 04 September 2002.

11. Diario Oficial de la Unión Europea. Versión consolidada del tratado de la Unión Europea. [online] 29.12.2006 [cited 2010 feb 21] Available from: URL: http://eur lex.europa.eu /LexUriServ/ LexUriServ.do? uri=OJ:C: 2006:321E:0001:0331:es:pdf

12. Sgreccia E. Manual de bioética. I Fundamentos y ética biomédica. Madrid: Biblioteca de Autores Cristianos; 2009: 226-227.

13. Utz AF. Ética social III. Barcelona: Herder; 1988.

14. Organización Mundial de la Salud. Promoción de la Salud, Glosario. [online] 1998 [cited 2011 mar 22] Available from: URL: http://www.bvs.org.ar/pdf/glosario_sp.pdf

15. Wallerstein N. What is the evidence on effectiveness of empowerment to improve health? Copenhagen: 
Aplicación del principio de subsidiariedad en el empoderamiento de los pacientes - Graciela Moya

WHO Regional Office for Europe; 2006. Health Evidence Network report. Available from: URL: http://www.euro.who.int/_data/assets/pdf_file/0010/74656/E88086.pdf

16. Aymé S, Kole A, Groft S. Empowerment of patients: Lessons from the rare disease community. Lancet 2008; 371: 2048-2051.

17. Taruscio D, Cerbo M. Rare diseases: general principles, specific problems, and health interventions. Ann Ist Super Sanita 1999: 35: 237-244.

18. Fundación GEISER [online] [cited 2009 sep 10] Available from: URL: http://fundaciongeiser.org/

19. Gericke CA, Riesberg A, Busse R. Ethical issues in funding orphan drug research and Development. Journal of Medical Ethics 2005; 31: 164-168.

20. Pellegrino ED, Thomasma DC. The virtues in Medical Practice. Oxford: Oxford University Press, 1993: 109-116.

21. Kole A, Faurisson F. (2009) The voice of 12.000 patients: experiences and expectations of rare disease patients on diagnosis and care in Europe. European Organisation for Rare Diseases (EURORDIS France) Available at http://www.eurordis.org/IMG/pdf/voice_12000_patients/EURORDISCARE_FULLBOOKr.pdf

22. NORD [online] [cited 2009 dic 19] Available from: URL: http://www.rarediseases.org

23. ORPHANED [online] [cited 2009 dic 19] Available from: URL: http://www.orpha.net

24. Remele K. The Good of All and of Each Individual: The Common Good, Communitarianism, and Catholic Public Philosophy [online] [cited 2011 feb 8] Available from: URL: www.uni-graz.at/ bibwww_soe_commongood.doc

25. McConkie-Rosell A, Sullivan J. Genetic Counseling-Stress, Coping, and the Empowerment Perspective. Journal of Genetic Counseling 1999; 8: 345-357.

Recibido: 8 de abril de 2012

Aceptado: 12 de junio de 2012 